\title{
Self-Organized UAV Flocking Based Only on Relative Range and Bearing
}

\author{
Thulio Guilherme Silva de Amorim, Tiago Pereira do Nascimento \\ Programa de Pós-Graduação em Informática \\ Centro de Informática - Universidade Federal da Paraíba \\ thulioguilherme53@gmail.com, tiagopn@ci.ufpb.br
}

\begin{abstract}
In this work, we address the problem of convergence and cohesiveness of an unmanned aerial vehicle (UAV) flocking. Thus, we propose a proximal control-based method for UAV self-organized flocking. Our method efficiently achieves flocking in the absence of alignment control and moves into an arbitrary direction without any direction control or informed robots. Robots use a Lennard-Jones potential function to maintain the cohesiveness of the flocking while avoiding collision within the teammates. We evaluate our method using the order metric, the steady-state value, and the settling time that can be used as a cohesiveness indicator.
\end{abstract}

Keywords: unmanned aerial vehicles; flocking; swarm robotics; self-organization.

\section{Introduction}

Flocking, also known as coordinated motion, is a behavior that consists of a large group of individuals moving together towards the same target direction. According to Ferrante et al. [1], flocking is traditionally realized using two main control rules: proximal control, which controls the cohesion of the swarm using local range-and bearing information about neighboring robots; and alignment control, which allows the robots to align in a common direction and uses more elaborate sensing mechanisms to obtain the orientation of neighboring robots.

Flocking can be achieved with the use of virtual physics-based design methods [2], where each robot is considered as a virtual particle that exerts virtual forces on other nearby robots. The most common methods for robot flocking usually use attraction-repulsion functions. Other works also add the alignment rule, e.g. the work of Ferrante et al. [1]. Some works even add information about a goal direction, e.g. the work of Tarcai et al. [3], and more recently Shirazi and Jin [4]. All of these methods have been applied to ground mobile robots, usually in an indoor environment, and some of them only in simulations.

UAV flocking algorithms are often tested only in simulations [5]. In contrast, recent fixed-wing UAV works have emerged with real robot experiments. Kownacki and Ołdziej [6], and Silic and Mohseni [7] have presented interesting studies, where the experiments rely on Global Positioning System (GPS) as the main positioning sensor and on communication between UAVs. Generally, UAV practical approaches use ranging systems based on radio signal transmissions [8]. Multi-rotor UAV flocking has also been tested in real conditions, the works of Virágh et al. [9] and Arul et al. [10] are example of contributions. Another recent work [11] has achieved flocking indoors with a swarm of up to 30 physical UAVs. This can be considered one of the most impressive achievements so far. Finally, all aerial mobile robot works cited uses an alignment control method which helps to achieve the flocking more quickly but requires more elaborate sensing mechanisms. In this work, we proposed a decentralized proximal control-based method for UAV self-organized flocking, which extends the work of Ferrante et al. [1].

\section{Methodology}

Flocking control methods are generally composed of three different term-functions: a proximal term, an alignment term, and an optional goal direction term, which is needed when the swarm is required to steer towards a specific target. Let us assume that in a flocking of $n$ robots, a robot $i$, with $i \in\{1, \ldots, n\}$, is called the focal robot. The focal robot computes a flocking control function denoted by $\mathcal{F}$. Taking into account all flocking control terms, the flocking control function can be calculated as follows:

$$
\mathcal{F}=\mathcal{P}+\mathcal{A}+\mathcal{G}
$$

where $\mathcal{P}$ is the proximal term, $\mathcal{A}$ is the alignment term, and $\mathcal{G}$ is the goal direction vector, which can only be used when there are informed robots (i.e. robots knowing the goal).

In this work, we propose a flocking control function $\mathcal{F}$, which needs only the proximal term to converge and move the UAVs into a unified direction. The equation (1) can therefore be rewritten as:

$$
\mathcal{F}=\mathcal{P} .
$$

The proximal term is used to make the UAV maintain the desired distance from other neighbor robots while keeping a cohesive formation. By using the proximal term, the focal robot can use the sensed range and bearing of its neighboring UAVs within a maximum interaction distance of $D_{p}$. For the model from our team's previous work [1] to be properly implemented on a flocking of UAVs, the distances between robots must be less than or equal to the maximum sensing range of the sensor. When the swarm converges into a stable formation, interactions between UAVs are limited to the first neighbors in the Voronoi sense, which in turn mandates that $D_{p}=\lambda d_{d e s}$, where $d_{d e s}$ is the desired distance between UAVs, and $\lambda$ is a positive gain that limits the maximum interaction distance to be less than 
twice the desired distance between two robots. Therefore, we propose the proximal term function as:

$$
\mathcal{P}=\sum_{i=1}^{m_{p}} p_{i}\left(d_{i}\right) e^{j \phi_{i}}
$$

where $m_{p}$ is the number of neighboring UAVs perceived by the focal robot within the maximum interaction distance, and $d_{i}$ and $\phi_{i}$ denote the relative range and bearing, both expressed in the body frame of reference of the focal robot.

The term $p_{i}\left(d_{i}\right)$ is the magnitude of the proximal vector. In our case, we employ the Lennard-Jones potential used by our team for unmanned ground vehicles (UGVs) [1], and it can be calculated as:

$$
p_{i}\left(d_{i}\right)=-\frac{4 \alpha \epsilon}{d_{i}}\left[2\left(\frac{\sigma}{d_{i}}\right)^{2 \alpha}-\left(\frac{\sigma}{d_{i}}\right)^{\alpha}\right]
$$

where $P\left(d_{i}\right)$ is a virtual potential function, $\epsilon$ is the strength of the potential function which determines its depth, $\alpha$ is the steepness of the potential function, and $\sigma$ is the amount of noise calculated by equation (5). Thus, the minimum of the potential function is when $d_{i}=-\epsilon$, and it can be expressed as:

$$
d_{i}=d_{\text {des }}=2^{\frac{1}{\alpha}} \sigma
$$

\section{Magnitude-dependent Flocking Motion Control}

The motion control is responsible for translates the output of the proximal control into a robot motion. The magnitude-dependent flocking motion control proposed by Ferrante et al. [1] makes possible the flocking in a random direction without an alignment control. In the following, we assume that the UAV has a translation axis $x$ and a rotational axis $y$. The desired linear translational movement $v$ is set as proportional to $f_{x}$, which is the projection of the flocking control vector $\mathcal{F}$ into the $x$ axis of the body reference frame, where the translational motion is defined according to the applied non-holonomic constraints. Conversely, the desired angular movement $\omega$ is set as proportional to $f_{x}$, the projection of $\mathcal{F}$ into the $y$ axis, where the rotational motion is defined according to the constraints.

We decompose the value of $\mathcal{F}$ into $f_{x}$ and $f_{y}$ by using the values of the bearing $\phi_{i}$. We call $f_{x}$ and $f_{y}$ the projection of the flocking control vector $\mathcal{F}$ on the XYplane of the body reference frame of the focal robot:

$$
\begin{aligned}
f_{x} & =\sum_{i=1}^{m_{p}} p_{i}\left(d_{i}\right) \cos \phi_{i} \\
f_{y} & =\sum_{i=1}^{m_{p}} p_{i}\left(d_{i}\right) \sin \phi_{i}
\end{aligned}
$$

where $\phi_{i}$ is the bearing.
Finally, the two movements can be calculated as:

$$
\begin{aligned}
& v=\kappa_{1} f_{x}+B_{s}, \\
& \omega=\kappa_{2} f_{y},
\end{aligned}
$$

where $B_{s}$ is the forward biasing movement, and $\kappa_{1}$ and $\kappa_{2}$ are the linear gains and the angular gains, respectively.

As the final step, we compute the desired pose for achieving or maintain the flocking using the linear movement $v$ and angular movement $\omega$ as described in the equation (8). To achieve the advantages of MDFMC, the desired pose is generated satisfying non-holonomic constraints:

$$
\begin{aligned}
& x_{d}(k)=x+v * \cos \theta, \\
& y_{d}(k)=y+v * \sin \theta, \\
& z_{d}(k)=z_{\text {coeff }}, \\
& \theta_{d}(k)=\theta+\omega,
\end{aligned}
$$

where $Z_{\text {coe } f f}$ is the desired height of the flock, $\left[x_{d}\right.$, $\left.y_{d}, z_{d}\right]^{T}=r_{d}$ is the desired position, and $\theta_{d}$ is the desired heading.

\section{Experiments}

In simulations, swarming algorithms usually are applied in a large group of robots ( $\geq 100$ ). When using ground robots, gathering this high number of robots is somehow feasible. However, the use of a large number of UAVs when simulating real disturbances from outdoor environments is not a simple task. Due to computational power limitations, we performed simulations with four robots but still succeeded in proving the efficiency of our proposed method.

\subsection{Used Metrics}

We use three metrics to analyze the effectiveness of our method. First, we use a metric that demonstrates how cohesive the swarm aligns in a common direction in a short period. This is the order metric $\psi$ which measures the degree of agreement of the orientations of the UAVs within the swarm. Thus, the vectorial sum of the headings of all $N$ robots is:

$$
\mathbf{b}=\sum_{i=1}^{N} e^{j \phi_{i}}
$$

and the order can be calculated as:

$$
\psi=\frac{1}{N}\|\mathbf{b}\|
$$

With this metric, the common heading is defined by a value of $\psi \approx 1$. When the UAVs point in different directions the value of $\psi \approx 0$. We also analyze the steady-state value that is reached for a given metric (in our case the order metric). The steady-state metric is the 
asymptotic value reached by the order metric during the simulation. We can compute the value of the steadystate as:

$$
\bar{\mu}=\frac{\sum_{(t=T-100)}^{T} \psi_{t}}{100}
$$

We also used the settling time which is the time needed to reach a steady-state in order. More precisely, the settling time $t^{*}$ is defined as the time for which $\forall t \geq t$ we have $\mu \geq 0.95 \bar{\mu}$. In other words, $t^{*}$ is the time at which and after which the order stays above the $95 \%$ of the steady-state.

\subsection{Results}

All the control and estimation are supported by the Multi-robot Systems (MRS) UAV system [12]. The MRS UAV system is an open-source implementation system for supporting replicable research through realistic simulations. We build our method using the Robot Operating System (ROS).

The simulation environment was implemented on the MRS simulator [12] which is a simulation environment based on the open-source Gazebo simulator. Similar to the real robot, the simulated multirotor UAV can estimate its pose through a GPS localization system and communicate with the others through Wi-Fi. Each UAV runs an instance of our proposed method and obtains the range $d$ and bearing $\phi$ of its neighboring by extracting it from the global position information of the others.

In the performed simulations, the initial position of each UAV was selected manually however the orientation was set randomly. After the take-off and hovering for a 10 s period, the simulation starts. We summarize the value of the parameters used in the simulation in Table 1.

Since there is no goal position to reach or any other kind of stopping criteria, we allowed the swarm to try to achieve and maintain the flocking for 240 seconds. Once this experiment duration was reached, the robots are triggered to finish the mission and standstill in their last position.

Table 1. Parameters values.

\begin{tabular}{c|c|c}
\hline Parameter & Description & Value \\
\hline$B_{S}$ & Maximum forward speed & $0.3 \mathrm{~m} / \mathrm{s}$ \\
\hline$\kappa_{1}$ & Linear gain & 0.5 \\
\hline$\kappa_{2}$ & Angular gain & 0.2 \\
\hline$\alpha$ & Steepness of potential function & 2 \\
\hline$\epsilon$ & Strength of potential function & 6 \\
\hline$d_{d e s}$ & Desired inter-robot distance & $6 \mathrm{~m}$ \\
\hline$D_{p}$ & Maximum interaction range & $10.8 \mathrm{~m}$ \\
\hline$\lambda$ & Interaction range gain & 1.8 \\
\hline$\gamma$ & Interpolation coefficient & 0.95 \\
\hline$z_{\text {coeff }}$ & Desired height & $2.5 \mathrm{~m}$ \\
\hline
\end{tabular}

With only the proximal method proposed, the UAVs rapidly converged in range and bearing as can be observed in the settling time of 26 seconds (see Figure 1). During the simulation, the flocking moved towards an arbitrary direction, as intended (see Figure 2). The simulation revealed a steady-state value of $\bar{\mu}=1.0097$ as it indicates that the order assumed values close to 1 during the simulation.

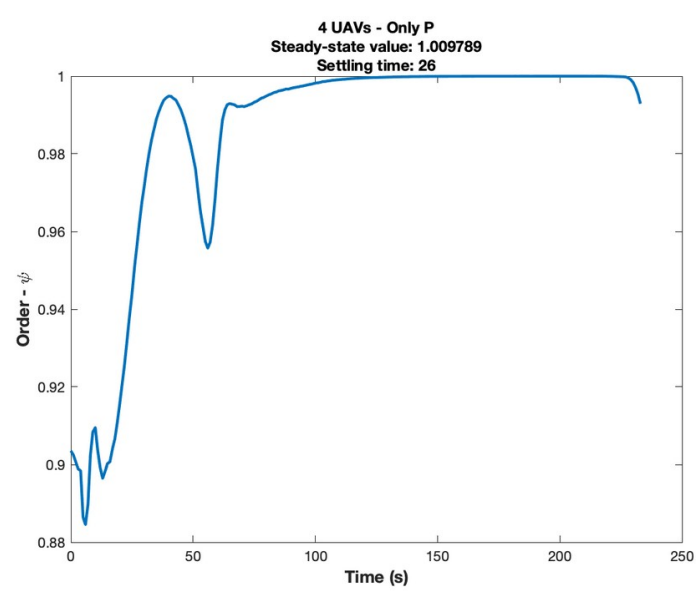

Figure 1. Order value in the experiment.

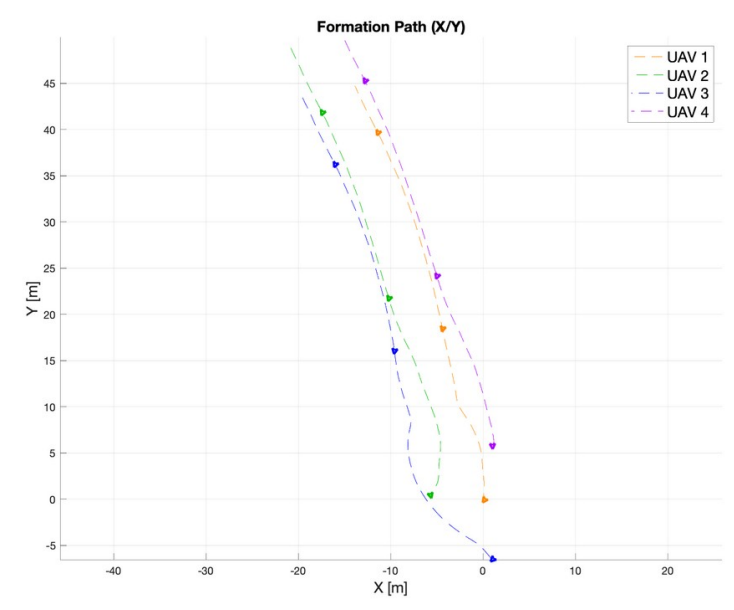

Figure 2. XY plot showing UAV headings during the simulation.

\section{Discussion}

The development of flocking algorithms in swarm robotics commonly uses ground robots in indoor environments mainly due to the simpleness of the environment setup. Basic research in the area of UAV flocking and formation flying was recently studied in [13]. Our results demonstrated that the proposed method works on UAVs. Furthermore, the simulations were performed in a distributed manner, where each robot has its control and flocking system. UAV swarm control is a relatively new field of research, and its applications are yet to be explored. Although, the adaptation of methods from ground robots to flying robots is usually mentioned as future work by many authors. We extend the work of Ferrante et al. [1] by adapting the decentralized proximal control-based method for UAV self-organized flocking. 


\section{Conclusion}

We have presented an efficient and simple method for UAV flocking based only on a proximal function. This function has been used to enable the UAVs not only to maintain a cohesive flock but to move in an arbitrary direction. We performed experiments using GPS and communication devices to exchange UAV positions and estimate the range and bearing. Our method managed to enable the robots to converge towards the flock and maintain the cohesiveness of the group. It also enabled the UAVs to move in an arbitrary direction. In this way, we achieved self-organized flocking for aerial robots using only proximal control. Simulations with a large number of robots and real-world experiments in outdoor environments are examples of future works.

\section{Bibliography}

[1] Ferrante, E.; Turgut, A. E.; Huepe, C.; Stranieri, A.; Pinciroli, C.; Dorigo, M. (2012) Self-organized flocking with a mobile robot swarm: A novel motion control method. Adaptive Behavior 20(6): 460-477. DOI: $\underline{10.1177 / 1059712312462248}$

[2] Brambilla, M.; Ferrante, E.; Birattari, M.; Dorigo, M. (2013) Swarm robotics: A review from the swarm engineering perspective. Swarm Intelligence 7:1-41. DOI: 10.1007/s11721-012-0075-2

[3] Tarcai, N.;Virágh, C.; Ábel, D.; Nagy, M.; Várkonyi, P. L.; Vásárhelyi, G.; Vicsek, T. (2011) Patterns, transitions and the role of leaders in the collective dynamics of a simple robotic flock. Journal of Statistical Mechanics: Theory and Experiment 2011(04): P04010. DOI: 10.1088/17425468/2011/04/p04010

[4] Shirazi, A. R.,; Jin, Y. (2020) Regulated morphogen gradients for target surrounding and adaptive shape formation. IEEE Transactions on Cognitive and Developmental Systems. DOI: 10.1109/TCDS.2020.2984087

[5] De Benedetti, M.; D’Urso, F.; Fortino, G.; Messina, F.; Pappalardo, G.; Santoro, C. (2017) A fault-tolerant selforganizing flocking approach for uav aerial survey. Journal of Network and Computer Applications 96: 14-30. DOI: $10.1016 /$ j.jnca.2017.08.004
[6] Kownacki, C. \& Ołdziej, D. (2016) Fixedwing uavs flock control through cohesion and repulsion behaviours combined with a leadership. International Journal of Advanced Robotic Systems 13(1): 36. DOI: $10.5772 / 62249$

[7] Silic, M. \& Mohseni, K. (2019) Field deployment of a plume monitoring uav flock. IEEE Robotics and Automation Letters 4(2): 769-775. DOI: 10.1109/LRA.2019.2893420

[8] Bhavana, T.; Nithya, M.; Rajesh, M. (2017) Leaderfollower coordination of multiple robots with obstacle avoidance. SmartTechCon, 613-617. DOI: 10.1109/SmartTechCon.2017.8358444

[9] Virágh, C.; Vásárhelyi, G.; Tarcai, N.; Szörényi, T.; Somorjai, G.; Nepusz, T.; Vicsek, T. (2014) Flocking algorithm for autonomous flying robots. Bioinspiration \& Biomimetics 9(2): 025012. DOI: $\quad$ 10.1088/17483182/9/2/025012

[10] Arul, S. H.; Sathyamoorthy, A. J.; Patel, S.; Otte, M.; Xu, H.; Lin, M. C.; Manocha, D. (2019) Lswarm: Efficient collision avoidance for large swarms with coverage constraints in complex urban scenes. IEEE Robotics and Automation Letters 4(4): 3940-3947. DOI: 10.1109/LRA.2019.2929981

[11] Vásárhelyi, G.; Virágh, C.; Somorjai, G.; Nepusz, T.; Eiben, A. E.; Vicsek, T. (2018) Optimized flocking of autonomous drones in confined environments. Science Robotics 3(20). DOI: 10.1126/scirobotics.aat3536

[12] Baca, T.; Petrlik, M.; Vrba, M.; Spurny, V.; Penicka, R.; Hert, D.; Saska, M. (2020) The MRS UAV System: Pushing the Frontiers of Reproducible Research, Realworld Deployment, and Education with Autonomous Unmanned Aerial Vehicles [arXiv: 2008.08050]. Retrieved November 9, 2020, from http://arxiv.org/abs/2008.08050

[13] Saska, M.; Hert, D.; Baca, T.; Kratky, V.; Nascimento, T. (2020) Formation Control of Unmanned Micro Aerial Vehicles for Straitened Environments. Autonomous Robots 44: 1573-7527. DOI: 10.1007/s10514-020-09913-0 